\title{
High Dietary $\omega-6: \omega-3$ PUFA Ratio Is Positively Associated with Excessive Adiposity and Waist Circumference
}

\author{
Nathaly Torres-Castillo ${ }^{a}$ Jorge Antonio Silva-Gómez ${ }^{a}$ Wendy Campos- \\ Perez $^{a} \quad$ Elisa Barron-Cabrera ${ }^{a}$ Ivan Hernandez-Cañaveral ${ }^{\text {b }}$ Mary Garcia- \\ Cazarin $^{c}$ Yolanda Marquez-Sandovald ${ }^{d}$ Karina Gonzalez-Becerra ${ }^{a}$ \\ Carlos Barron-Gallardo ${ }^{a}$ Erika Martinez-Lopez ${ }^{a}$ \\ a Medical Molecular Biology Service, "Fray Antonio Alcalde" Civil Hospital of Guadalajara, \\ Department of Molecular Biology and Genomics, University Center of Health Sciences, \\ University of Guadalajara, Guadalajara, Mexico; ${ }^{b}$ Department of Microbiology and \\ Parasitology, University Center of Health Sciences, University of Guadalajara, Guadalajara, \\ Mexico; ${ }^{c}$ Office of Disease Prevention, National Institutes of Health, Bethesda, MD, USA; \\ dDepartment of Human Reproduction and Child Growth and Development, University \\ Center of Health Sciences, University of Guadalajara, Guadalajara, Mexico
}

Keywords

Polyunsaturated fatty acids · Omegas · Obesity · Adiposity $\cdot$ Adiponectin

\begin{abstract}
Objective: The aim of this study was to analyze dietary $\omega-6: \omega-3$ polyunsaturated fatty acid (PUFA) ratio and its association with adiposity and serum adiponectin levels in a Mexican population. Methods: In this cross-sectional study, individuals with a BMI $\geq 18.5 \mathrm{~kg} / \mathrm{m}^{2}$, were classified using four methods to measure adiposity. Parameters of body composition were measured by InBody 3.0. Diet intake was evaluated prospectively using a 3-day written food record. Serum high-molecular weight adiponectin isoform was measured using an ELISA assay. Biochemical and adiposity variables were analyzed by tertiles of dietary $\omega-6: \omega-3$ PUFA ratio. Results: A total of 170 subjects were recruited with a mean age of $36.9 \pm 11.8$ years. The $73.5 \%$ of subjects were women. Subjects in the higher tertile of dietary $\omega-6: \omega-3$ PUFA ratio had more adiposity and higher levels of triglycerides, VLDL-c, glucose, insulin and HOMA-IR than those in the first tertile $(p<0.05)$. Adiponectin levels showed a trend according to dietary
\end{abstract}

Nathaly Torres-Castillo and Jorge Antonio Silva-Gómez contributed equally to this study.

Erika Martinez-Lopez, PhD

Medical Molecular Biology

'Fray Antonio Alcalde' Civil Hospital of Guadalajara

44280 Guadalajara, Jalisco, Mexico

erikamtz27@yahoo.com.mx 
$\omega-6: \omega-3$ PUFA ratio $(p=0.06)$. A linear regression model showed that waist circumference, insulin, and HOMA-IR have positive associations with dietary $\omega-6: \omega-3$ PUFA ratio. Conclusion: This study suggests that high dietary $\omega-6: \omega-3$ PUFA ratio is positively associated with excessive adiposity and worse metabolic profile.

(C) 2018 The Author(s)

Published by S. Karger GmbH, Freiburg

\section{Introduction}

Obesity is an abnormal accumulation of fat mass that can be harmful to health. The prevalence of overweight and obesity has increased by more than $50 \%$ worldwide since 1980 [1]. Data from the ENSANUT (Encuesta Nacional de Salud y Nutrición) 2016 survey showed that $38.6 \%$ of women and $27.7 \%$ of men in Mexico are obese [2].

This condition is one of the main risk factors for the development of non-communicable chronic diseases (NCDs) [3], such as hypertension, ischemic heart disease, stroke and type 2 diabetes mellitus (T2DM), which cause approximately $63 \%$ of the total number of deaths worldwide $[4,5]$.

High-molecular-weight (HMW) adiponectin isoform is an adipocytokine secreted by adipocytes; it has been shown to have anti-inflammatory and anti-atherogenic properties, while hypoadiponectinemia has been associated with insulin resistance, T2DM, and cardiovascular disease [6, 7]. This adipocytokine is expressed through the peroxisome proliferatoractivated receptor gamma (PPAR $\gamma$ ), which is activated by $\omega-3$ polyunsaturated fatty acid (PUFA) [7].

Overfeeding leads to adiposity, defined as body fat accumulation, which in turn leads to adipocyte dysfunction [8]. In this state, lower levels of adiponectin are observed [9]. However, the degree to which an inappropriate intake of macronutrients, and particularly a high dietary $\omega-6: \omega-3$ PUFA ratio, may be associated with adiposity and abnormal adiponectin levels remains unclear [10].

Excess adiposity is accompanied by a chronic state of low-grade inflammation [11]. The $\omega-6$ PUFAs are precursors of pro-inflammatory eicosanoids, while $\omega$-3 PUFAs produce more anti-inflammatory eicosanoids, cytokines, and resolvins [12]. Moreover, it has been reported that the intake of $\omega$-3 PUFAs in Western countries is insufficient and often accompanied by a dietary $\omega-6: \omega-3$ PUFA ratio higher than $10: 1[13,14]$. Thus, the objective of this study was to determine the dietary $\omega-6: \omega-3$ PUFA ratio and its association with adiposity and serum adiponectin levels in a Mexican population.

\section{Material and Methods}

\section{Subjects}

A total of 256 subjects aged 18-65 years (70 male and 186 female) with a BMI $\geq 18.5 \mathrm{~kg} / \mathrm{m}^{2}$ were considered for the study. Subjects were recruited via posters and leaflets. A total of 86 subjects were excluded due to incomplete data (fig. 1). The trial was conducted from May 2010 to December 2014 in Guadalajara, Jalisco, Mexico. Measurements were taken at the Medical Molecular Biology Service at the 'Fray Antonio Alcalde' Civil Hospital of Guadalajara. Exclusion criteria included pregnancy, alcoholism (more than $20 \mathrm{~g} /$ day and $40 \mathrm{~g}$ /day of ethanol in women and men, respectively), history of cardiovascular disease or stroke, endocrine diseases, T2DM, or any other chronic degenerative disease. None of the subjects were tobacco, and/or $\omega$-3 supplement users, and none were taking lipid or weight loss medication, sulfonylureas, thiazolidinediones, insulin, glucocorticoids, antineoplastic agents, angiotensin receptor blockers, angiotensin-converting enzyme inhibitors, or psychoactive medications. 
Torres-Castillo et al.: High Dietary $w-6: \omega-3$ PUFA Ratio Is Positively Associated with Excessive Adiposity and Waist Circumference

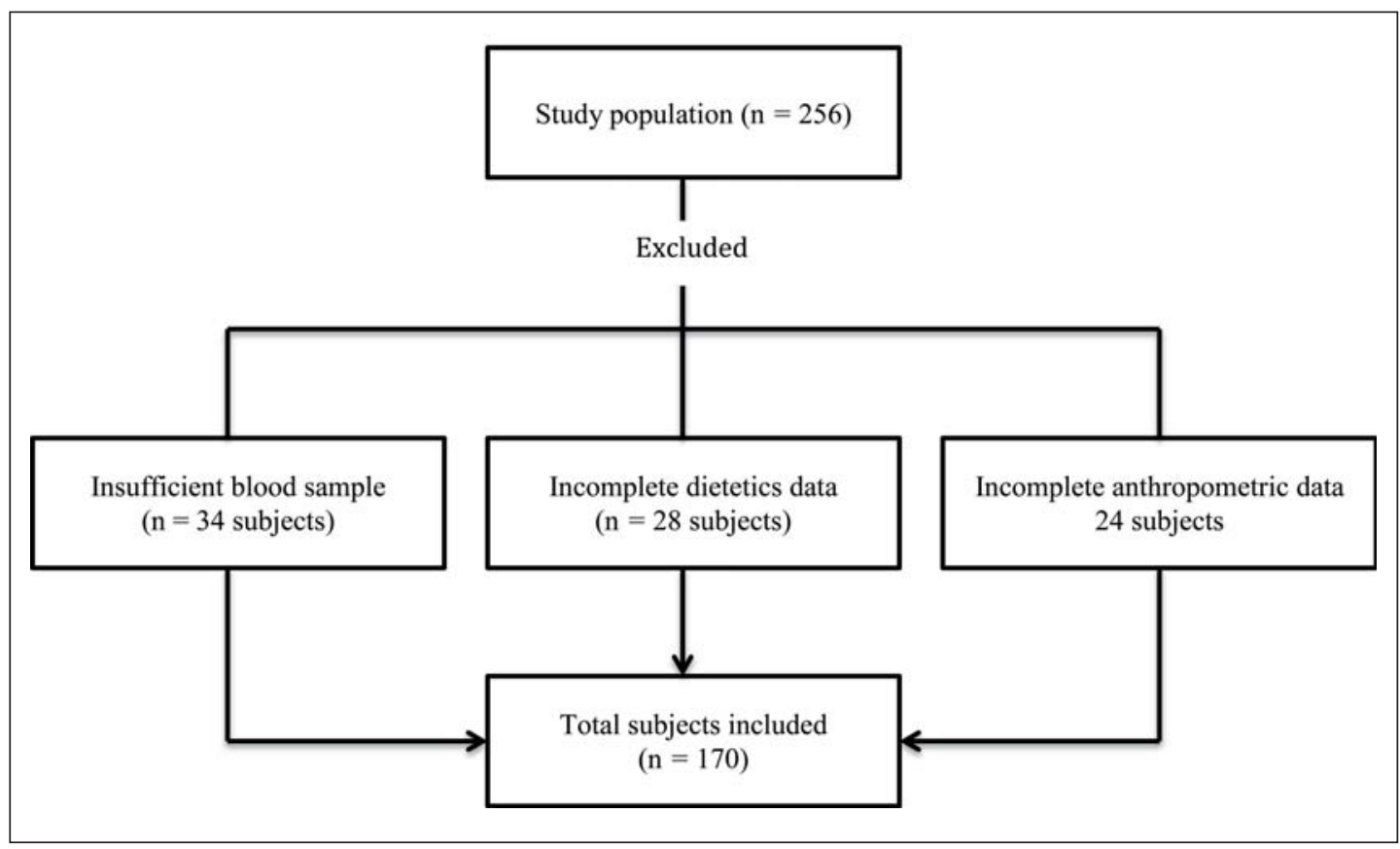

Fig. 1. Flow chart of the study population.

Ethics

Written informed consent was obtained from all participants, and all information was coded to keep the name of the subjects confidentially. The study was approved by the Hospital's Ethics Committee and by the University of Guadalajara's Human Research Ethics Committee (Registration number: CI/019/2010) and was realized according to the Declaration of Helsinki in its revised version of 2013 [15].

\section{Dietary Intake}

Subjects were provided with a prospective serial assessment of nutritional intake using a 3-day written food record. All were instructed on how to record the type, amount, and preparation methods of the food they consumed during 2 weekdays and 1 weekend day. Dietary data were assessed using food scales and models to increase the accuracy of reported portion sizes. The food records were reviewed and coded by a trained registered nutritionist. Nutritional intake was calculated using Nutritionist Pro ${ }^{\text {TM }}$ Diet Analysis software (Axxya Systems, Stafford, TX, USA). Newer Mexican foods that were not listed in the Nutrient Composition of Mexican Food Guidelines were added to the Nutritionist Pro database.

\section{Anthropometric Measurements}

Anthropometric and clinical parameters were measured following a 12-hour fast and after having evacuated bowels. Height and weight were measured with subjects wearing a provided disposable gown. BMI was calculated as weight in kilograms divided by height in meters squared. Waist circumference (WC) (narrowest diameter between xiphoid process and iliac crest) was measured using a Lufkin measuring tape. Tetrapolar body electrical bioimpedance was used to determine body composition including protein and fat mass (InBody 3.0 body composition analyzer; Biospace Co Ltd, Seoul, South Korea). Body adiposity index (BAI) was calculated using the formula (hip circumference) / ((height) × 1.5) -18 [16].

\section{Definition of Excess Adiposity}

While BMI is one of the most useful methods for the diagnosis of obesity, it measures only total body mass and does not reflect adipose tissue mass. In order to ensure a more optimal approach to adiposity and its relationship with the variables of interest, we also used body fat percentage (BFP), WC, and BAI the latter of which is a new adiposity measurement method [16]. The cutoffs used to determine excess adiposity were $\mathrm{BMI} \geq 30 \mathrm{~kg} / \mathrm{m}^{2}$ in both men and women, BFP $>20 \%$ in men and $>30 \%$ in women, $\mathrm{WC} \geq 90 \mathrm{~cm}$ in men and $\geq 80 \mathrm{~cm}$ women, and BAI > 21\% in men and > 33\% in women [17]. 
Torres-Castillo et al.: High Dietary $\omega-6: \omega-3$ PUFA Ratio Is Positively Associated with Excessive Adiposity and Waist Circumference

\section{Biochemical Measurements}

Venous blood samples were taken and immediately centrifuged to obtain the serum. The samples were then analyzed and frozen at $-80^{\circ} \mathrm{C}$ for preservation. All biochemical evaluations were performed simultaneously (in order to minimize analytical variability) using a Vitros-250 dry chemistry analyzer (Ortho-Clinical Diagnostics, Johnson \& Johnson Services, Inc., Rochester, NY, USA). The following tests were performed: glucose, triglycerides, cholesterol, high-density lipoprotein cholesterol (HDL-c) and C-reactive protein (CRP). Low-density lipoprotein cholesterol (LDL-c) was calculated using the Friedewald formula [18] excluding subjects with serum triglyceride values above $400 \mathrm{mg} / \mathrm{dl}$ [19]. Concentration of very-low-density lipoprotein cholesterol (VLDL-c) was calculated as VLDL-c = total cholesterol - (LDL-c + HDL-c).

For quality control purposes, a human pooled serum and a commercial control serum (Ortho-Clinical Diagnostics) were used to account for imprecision and inaccuracy of the biochemical measurements. The intra-assay variability (CV\%) of biochemical assays was relative to 10 repeated determinations of the control serum in the same analytical session, whereas inter-assay CV\% for each variable was calculated from the mean values of control sera measured over five analytical sessions. Insulin levels were determined using an ELISA assay (Monobind Inc, Lake Forest, CA, USA) according to instructions from the supplier. Insulin resistance was estimated according to the homeostasis model assessment of insulin resistance (HOMA-IR) [20] and calculated as follows: (Fasting insulin $(\mu \mathrm{U} / \mathrm{ml}) \times$ fasting glucose $(\mathrm{mg} / \mathrm{dl})$ ) / 405, where HOMA-IR > 2.5 indicated the presence of insulin resistance.

\section{Adiponectin}

HMW adiponectin isoform was measured by ELISA assay according to the instructions from the supplier (ALPCO Adiponectin ELISA, 2013). The recovery rate was almost 100\%, and the intra- and inter-assay coefficients of variation for adiponectin were 3.3\% and 7.4\%, respectively (ALPCO Diagnostics, Salem, NH, USA). Normal serum HMW adiponectin levels were considered to be $3 \mu \mathrm{g} / \mathrm{ml}$ [21].

\section{Statistical Analyses}

The Kolmogorov-Smirnov test was used to determine normal distribution of the variables. Student's $\mathrm{t}$ and Mann-Whitney $\mathrm{U}$ tests were used to analyze the differences in demographic, anthropometric, biochemical, and nutritional characteristics of the study population by sex. Because adiposity measurements display sexual dimorphism, analysis in this study were adjusted by sex. ANCOVA test was used to test differences in biochemical and adiposity variables according to tertiles of dietary $\omega-6: \omega-3$ PUFA ratio, with energy intake and sex as covariates. Energy-adjusted intake was calculated by adding the mean of PUFA intake to the residual from the linear regression model. Multivariable linear regression analysis was performed to identify the associations of dietary $\omega-6: \omega-3$ PUFA ratio with biochemical and adiposity variables. All models were adjusted for age, sex, energy intake and weight, except for the association of BMI, in which weight was not consider as covariate in order to avoid collinearity. Statistical analyses were performed using SPSS v.20.0 (IBM, Armonk, NY, USA), and a p value $<0.05$ was considered statistically significant.

\section{Results}

\section{Population Description}

A total of 170 subjects were included. The $73.5 \%$ were women $(n=125)$. According to the four methods used for adiposity classification, more than $60 \%$ of the study population presented excessive adiposity. Weight, BFP, protein mass, adiponectin, and energy intake varied by sex, as expected. Dietary $\omega-6$ and $\omega-3$ PUFA intake were higher in men than in women, but the dietary $\omega-6: \omega-3$ PUFA ratio was identical in both sexes. Additional characteristics are presented in table 1.

\section{Dietary $\omega-6$ and $\omega-3$ PUFAs}

The mean of dietary $\omega-6$ PUFA was $15.9 \pm 10.7 \mathrm{~g}$, and it was obtained by adding $\alpha$-linoleic acid (18:2) and arachidonic acid (AA, 20:4). The intake of $\omega$-3 PUFA was $1.5 \pm 0.9 \mathrm{~g}$, and it was calculated using the amount of $\alpha$-linolenic acid (ALA, 18:3), stearidonic acid (18:4), eicosa- 
Torres-Castillo et al.: High Dietary $\omega-6: \omega-3$ PUFA Ratio Is Positively Associated with Excessive Adiposity and Waist Circumference

Table 1. Demographic, anthropometric, biochemical and nutritional characteristics of the study population

\begin{tabular}{|c|c|c|c|c|}
\hline Variable & $\begin{array}{l}\text { All } \\
(n=170)\end{array}$ & $\begin{array}{l}\text { Women } \\
(n=125)\end{array}$ & $\begin{array}{l}\text { Men } \\
(n=45)\end{array}$ & $\mathrm{p}$ value \\
\hline \multicolumn{5}{|c|}{ Demographic and anthropometric variables } \\
\hline Age, years & $36.9 \pm 11.8^{1}$ & $36.7 \pm 11.8$ & $37.5 \pm 11.9$ & 0.687 \\
\hline Weight, kg & $76.3 \pm 19.2$ & $73.9 \pm 18.5$ & $83.0 \pm 19.4$ & 0.006 \\
\hline BMI, $\mathrm{kg} / \mathrm{m}^{2}$ & $28.6 \pm 7.0$ & $29.0 \pm 7.4$ & $27.4 \pm 5.6$ & 0.171 \\
\hline Body fat, $\%$ & $33.7 \pm 9.5$ & $36.4 \pm 8.3$ & $24.8 \pm 6.9$ & $<0.001$ \\
\hline Body fat, kg & $26.5 \pm 13.4$ & $28.0 \pm 13.4$ & $22.5 \pm 12.7$ & 0.019 \\
\hline Protein mass, kg & $12.5 \pm 2.6$ & $11.5 \pm 1.7$ & $15.5 \pm 2.6$ & $<0.001$ \\
\hline Waist circumference, $\mathrm{cm}$ & $91.9 \pm 17.2$ & $90.5 \pm 17.7$ & $95.8 \pm 15.0$ & 0.071 \\
\hline Hip circumference, cm & $106.2 \pm 13.6$ & $107.4 \pm 14.4$ & $103.2 \pm 10.6$ & 0.079 \\
\hline \multicolumn{5}{|l|}{ Biochemical variables } \\
\hline Cholesterol, mg/dl & $187.6 \pm 36.0$ & $189.3 \pm 35.4$ & $183.2 \pm 37.5$ & 0.332 \\
\hline HDL-c, mg/dl & $44.1 \pm 11.5$ & $46.0 \pm 11.9$ & $39.0 \pm 8.4$ & $<0.001$ \\
\hline LDL-c, mg/dl & $115.6 \pm 32.7$ & $116.2 \pm 31.8$ & $114.0 \pm 35.4$ & 0.709 \\
\hline VLDL-c, mg/dl & $27.6 \pm 14.0$ & $26.7 \pm 13.2$ & $30.1 \pm 15.9$ & 0.168 \\
\hline Triglycerides, mg/dl & $140.8 \pm 72.8$ & $134.4 \pm 65.4$ & $158.4 \pm 88.7$ & 0.101 \\
\hline Glucose, mg/dl & $89.5 \pm 10.4$ & $89.5 \pm 10.5$ & $89.4 \pm 10.3$ & 0.474 \\
\hline Insulin, $\mu \mathrm{U} / \mathrm{ml}$ & $12.1 \pm 8.3$ & $11.4 \pm 7.4$ & $13.7 \pm 10.2$ & 0.216 \\
\hline HOMA-IR & $2.7 \pm 2.1$ & $2.8 \pm 2.1$ & $3.3 \pm 3.0$ & 0.196 \\
\hline Adiponectin HMW, $\mu \mathrm{g} / \mathrm{ml}$ & $3.2 \pm 2.1$ & $3.6 \pm 2.3$ & $2.1 \pm 1.1$ & $<0.001$ \\
\hline \multicolumn{5}{|l|}{ Nutritional variables } \\
\hline Energy, kcal & $2,413.7 \pm 1,018.7$ & $2,222.9 \pm 841.5$ & $3,032.9 \pm 1,277.0$ & $<0.001$ \\
\hline Protein, \% & $17.1 \pm 3.9$ & $17.0 \pm 4.0$ & $17.4 \pm 3.8$ & 0.582 \\
\hline Carbohydrates, \% & $47.1 \pm 8.7$ & $47.7 \pm 8.5$ & $45.2 \pm 9.1$ & 0.069 \\
\hline Fat, \% & $35.4 \pm 8.2$ & $35.0 \pm 8.0$ & $36.7 \pm 8.4$ & 0.188 \\
\hline SFA, $\%$ & $11.3 \pm 3.8$ & $11.2 \pm 3.6$ & $11.6 \pm 4.5$ & 0.469 \\
\hline MUFA, $\%$ & $10.2 \pm 3.4$ & $10.2 \pm 3.3$ & $10.5 \pm 3.8$ & 0.555 \\
\hline PUFA, \% & $7.3 \pm 4.0$ & $7.3 \pm 4.2$ & $7.2 \pm 3.2$ & 0.699 \\
\hline Cholesterol, mg & $301.2 \pm 241.4$ & $257.1 \pm 207.0$ & $444.3 \pm 287.9$ & $<0.001$ \\
\hline$\omega 6, \mathrm{~g}$ & $15.9 \pm 10.7$ & $14.2 \pm 9.2$ & $20.8 \pm 13.1$ & $<0.001$ \\
\hline$\omega 3, \mathrm{~g}$ & $1.5 \pm 0.9$ & $1.3 \pm 0.8$ & $1.8 \pm 1.0$ & 0.001 \\
\hline$\omega 6: \omega 3$ ratio & $14: 1$ & $11: 1$ & $13: 1$ & 0.311 \\
\hline Fiber, $g$ & $25.9 \pm 13.2$ & $24.8 \pm 10.8$ & $29.4 \pm 18.8$ & 0.045 \\
\hline sHC, $\%$ & $27.1 \pm 13.6$ & $27.9 \pm 13.3$ & $24.2 \pm 14.4$ & 0.082 \\
\hline Alcohol, g & $1.8 \pm 5.9$ & $1.2 \pm 4.4$ & $3.7 \pm 9.2$ & 0.092 \\
\hline \multicolumn{5}{|c|}{ Methods of determining excessive adiposity } \\
\hline Body fat percentage, $\%$ & $77.1^{2}$ & 77.6 & 75.6 & 0.837 \\
\hline Body adiposity index, \% & 59.8 & 47.9 & 91.1 & $<0.001$ \\
\hline BMI, \% & 62.9 & 65.6 & 55.6 & 0.281 \\
\hline Waist circumference, $\%$ & 65.9 & 67.2 & 62.2 & 0.585 \\
\hline
\end{tabular}

p = Probability with t-Student or Mann-Whitney U test; HMW = high molecular weight; SFA = saturated fatty acids; MUFA = monounsaturated fatty acids; PUFA = polyunsaturated fatty acids; $\mathrm{sHC}=$ simple carbohydrates.

${ }^{1}$ Mean \pm SD, ${ }^{2}$ Percentage $(\%)$. 
Torres-Castillo et al.: High Dietary $\omega-6: \omega-3$ PUFA Ratio Is Positively Associated with Excessive Adiposity and Waist Circumference

Table 2. Comparison of biochemical variables by tertiles of dietary $\omega-6: \omega-3$ PUFA ratio

\begin{tabular}{lllll}
\hline & $\begin{array}{l}\text { Tertile } 1 \\
\omega-6: \omega-3\end{array}$ & $\begin{array}{l}\text { Tertile } 2 \\
\omega-6: \omega-3\end{array}$ & $\begin{array}{l}\text { Tertile 3 } \\
\omega-6: \omega-3\end{array}$ & p value \\
& $\begin{array}{l}2.39: 1 \\
(\mathrm{n}=56)\end{array}$ & $\begin{array}{l}\geq 8.39: 1-\leq 11.74: 1 \\
(\mathrm{n}=57)\end{array}$ & $\begin{array}{l}>11.74: 1 \\
(\mathrm{n}=58)\end{array}$ & \\
\hline Total cholesterol, mg/dl & $181.9 \pm 5.0$ & $188.7 \pm 4.8$ & $192.0 \pm 4.8$ & 0.364 \\
HDL-c, mg/dl & $46.6 \pm 1.5$ & $43.3 \pm 1.4$ & $42.4 \pm 1.5$ & 0.134 \\
LDL-c, mg/dl & $109.9 \pm 4.7$ & $117.8 \pm 4.5$ & $118.9 \pm 4.4$ & 0.346 \\
VLDL-c, mg/dl & $24.4 \pm 2.0^{\mathrm{a}}$ & $26.2 \pm 1.9^{\mathrm{a}, \mathrm{b}}$ & $31.8 \pm 1.8^{\mathrm{b}}$ & 0.019 \\
Triglycerides, mg/dl & $126.8 \pm 10.0^{\mathrm{a}}$ & $133.5 \pm 9.4^{\mathrm{a}, \mathrm{b}}$ & $161.1 \pm 9.5^{\mathrm{b}}$ & 0.035 \\
Glucose, mg/dl & $86.2 \pm 1.4^{\mathrm{a}}$ & $90.5 \pm 1.4^{\mathrm{a}, \mathrm{b}}$ & $91.6 \pm 1.4^{\mathrm{b}}$ & 0.023 \\
Insulin, $\mu \mathrm{d} / \mathrm{ml}$ & $7.7 \pm 1.4^{\mathrm{a}}$ & $13.9 \pm .5^{\mathrm{b}, \mathrm{c}}$ & $15.0 \pm 1.4^{\mathrm{c}}$ & 0.001 \\
HOMA-IR & $2.1 \pm 0.3^{\mathrm{a}}$ & $3.0 \pm 0.3^{\mathrm{a}, \mathrm{b}}$ & $3.7 \pm 0.3^{\mathrm{b}}$ & 0.002 \\
Adiponectin HMW, $\mu \mathrm{mg} / \mathrm{ml}$ & $3.7 \pm 0.3$ & $3.0 \pm 0.3$ & $2.9 \pm 0.3$ & 0.061 \\
\hline
\end{tabular}

$\mathrm{TC}=$ Total cholesterol; HOMA-IR = homeostatic model assessment of insulin resistance; HMW = high molecular weight.

Values are shown as mean and standard error. Data were adjusted by sex and energy intake. Means with the same letter subscripts are equal. Means with different letter subscripts are different.

Table 3. Comparison of adiposity measurements by tertiles of dietary $\omega-6: \omega-3$ PUFA ratio

\begin{tabular}{|c|c|c|c|c|}
\hline & $\begin{array}{l}\text { Tertile } 1 \\
\omega-6: \omega-3 \\
<8.39: 1 \\
(n=56)\end{array}$ & $\begin{array}{l}\text { Tertile } 2 \\
\omega-6: \omega-3 \\
\geq 8.39: 1-\leq 11.74: 1 \\
(n=57)\end{array}$ & $\begin{array}{l}\text { Tertile } 3 \\
\omega-6: \omega-3 \\
>11.74: 1 \\
(n=58)\end{array}$ & $p$-value \\
\hline Body fat percentage, $\%$ & $30.7 \pm 1.1^{\mathrm{a}}$ & $34.5 \pm 1.0^{\mathrm{b}}$ & $34.7 \pm 1.0^{\mathrm{b}}$ & 0.015 \\
\hline Body adiposity index, \% & $31.4 \pm 1.0$ & $34.0 \pm 1.0$ & $33.4 \pm 1.0$ & 0.172 \\
\hline $\mathrm{BM}, \mathrm{kg} / \mathrm{m}^{2}$ & $26.7 \pm 1.0$ & $29.9 \pm 0.9$ & $29.1 \pm 0.9$ & 0.053 \\
\hline Waist circumference, $\mathrm{cm}$ & $87.6 \pm 2.4$ & $93.7 \pm 2.2$ & $94.1 \pm 2.3$ & 0.098 \\
\hline
\end{tabular}

Values are shown as mean and standard error. Data were adjusted by sex and energy intake. Means with the same letter subscripts are equal. Means with different letter subscripts are different.

pentaenoic acid (EPA, 20:5), and docosahexaenoic acid (DHA, 22:6). Hence, $\omega-6$ and $\omega-3$ PUFA intake were used to calculate the dietary $\omega-6: \omega-3$ PUFA ratio, the result of which was $14: 1$.

\section{Tertiles of Dietary $\omega-6: \omega-3$ PUFA Ratio and Biochemical Variables}

Biochemical and adiposity measurements were analyzed by tertiles of dietary $\omega-6: \omega-3$ PUFA ratio. Subjects in the higher tertile of dietary $\omega-6: \omega-3$ PUFA ratio had higher VLDL-C, triglycerides, glucose, insulin, and HOMA-IR than subjects in the lowest tertile. Besides, adiponectin levels showed a considerable trend toward significance with lower levels in the third tertile and higher levels in first tertile of dietary $\omega-6: \omega-3$ PUFA ratio $(p=0.061)$. Other biochemical variables compared by tertiles showed no statistical differences (table 2).

\section{Tertiles of Dietary $\omega-6: \omega-3$ PUFA Ratio and Adiposity}

Related to the four adiposity measurements, only BFP showed differences between tertiles of dietary $\omega-6: \omega-3$ PUFA ratio. Subjects in third tertile presented higher BFP than 
Torres-Castillo et al.: High Dietary $\omega-6: \omega-3$ PUFA Ratio Is Positively Associated with Excessive Adiposity and Waist Circumference

Table 4. Association of dietary $\omega-6: \omega-3$ PUFA ratio (independent variable) with adiposity and biochemical variables in the study population $(n=170)$

\begin{tabular}{lllll}
\hline Model* $^{*}$ & Dependent variable & $\mathrm{R}^{2}, \%$ & $\mathrm{~B}(95 \% \mathrm{CI})$ & $\mathrm{p}$ value \\
\hline Model 1 & WC, $\mathrm{cm}$ & 86.6 & $0.228(0.041-0.415)$ & 0.017 \\
Model 2 & Insulin, $\mu \mathrm{U} / \mathrm{ml}$ & 37.9 & $0.276(0.037-0.514)$ & 0.024 \\
Model 3 & HOMA-IR & 23.4 & $0.100(0.040-0.161)$ & 0.001 \\
\hline
\end{tabular}

WC = Waist circumference; HOMA-IR = homeostatic model assessment of insulin resistance.

*All models were adjusted by age, sex, weight and energy intake.

those in the first tertile. There was a trend towards significance in BMI $(p=0.05)$ and WC $(p=0.09)$, in which subjects in the third tertile had the higher adiposity values (table 3 ).

\section{Association of Dietary $\omega-6: \omega-3$ PUFA Ratio with Adiposity and Biochemical Variables}

A multivariable linear regression analysis was conducted to test for the presence of an association of dietary $\omega-6: \omega-3$ PUFA ratio with adiposity and biochemical variables. All adiposity and biochemical variables that showed a tendency or were significant in the univariable analysis were selected to test an association in the multivariable model. In that sense, WC, insulin, and HOMA-IR, were positively associated with dietary $\omega-6: \omega-3$ PUFA ratio (table 4). On the other hand, VLDL-c, triglycerides, glucose, and adiponectin levels did not present any significant difference (data not shown).

\section{Discussion}

Due to the relationship between excessive fat consumption and the development of obesity, greater attention has been focused on the intake of certain types of fatty acids and their relationship with adiposity, as well as on alterations in different molecules secreted by adipose tissue, such as adiponectin. In this study, we focused on the dietary $\omega-6: \omega-3$ PUFA ratio, and the ratio of $14: 1$ detected in our study is consistent with other studies reporting a ratio of 16:1 [22]. It has been suggested that this elevated ratio causes greater and chronic inflammation status, which in turn leads to a vicious cycle in which low-grade inflammation favors obesity and vice versa [23-25].

In addition, subjects with a low dietary $\omega-6: \omega-3$ PUFA ratio (first tertile) had better triglyceride, VLDL-c, glucose, insulin, and HOMA-IR parameters than those with a higher dietary ratio (third tertile). Besides, in a multivariable model, insulin and HOMA-IR were positively associated with the dietary $\omega-6: \omega-3$ PUFA ratio. In vivo studies have shown that mice that were fed with a high dietary $\omega-6: \omega-3$ PUFA ratio had higher levels of triglycerides in comparison with those fed with the lowest ratio [26]. Likewise, when rats were fed with a diet with low $\omega-6: \omega-3$ PUFA ratio, glucose and insulin levels improved; moreover, in some cases these changes were accompanied by lower levels of pro-inflammatory cytokines [27]. Human studies have shown that subjects who receive a dietary supplementation with $\omega-3$ PUFAs, decrease their AA:EPA ratio, and this change is accompanied by a reduction in triglyceride levels that is not observed in subjects receiving placebo treatments [28].

Most human studies related to dietary PUFAs, only report the intake of $\omega-3$ PUFAs, but not the $\omega-6: \omega-3$ PUFA ratio. However, it is expected that an increase in the intake of $\omega-3$ PUFAs will be followed by a decrease in the dietary $\omega-6: \omega-3$ PUFA ratio [29]. In that regard, it has been reported that subjects who increased their consumption of $\omega-3$ PUFAs showed 
lower levels of glucose, insulin, HOMA-IR, and triglycerides as well as higher levels of HDL-c and adiponectin. This finding is consistent with the results of the present study [30]. In our study, adiponectin levels showed a trend according to dietary $\omega-6: \omega-3$ PUFA ratio $(p=0.06)$. It has been suggested that the association between adiponectin and $\omega-3$ PUFAs is linked with a better inflammatory profile and lower levels of tumor necrosis factor- $\alpha$, which has been associated with lower adiponectin levels [31]. However, more studies are necessary in order to elucidate this mechanism.

Regarding adiposity measurements, the subjects in the present study had higher BFP when a higher dietary $\omega-6: \omega-3$ PUFA ratio was found compared to individuals who consumed the lowest dietary ratio (first tertile). Nevertheless, this was not confirmed in the multivariable model. In contrast, Lund et al. [32] observed a negative association between dietary $\omega-3$ PUFAs and BFP, but no association was found between dietary $\omega-6: \omega-3$ PUFA ratio and WC or BFP. This finding may be attributable to a ratio of dietary $\omega-6: \omega-3$ PUFAs of $4: 1$, a ratio considered to be optimal. Similarly, it has been reported that subjects who consume more $\omega-3$ PUFAs over a 10-week period present lower body fat mass than those in a control group [33]. On the other hand, in present study, a positive association of dietary $\omega-6: \omega-3$ PUFA ratio with WC was found. It has been reported that higher intake of $\omega-3$ PUFAs was associated with lower prevalence of enlarged WC [34]. In vitro, $\omega-6$ PUFAs have been shown to favor the accumulation of triglycerides in adipose tissue [35], and $\omega$-3 PUFAs were a negative regulator of lipogenesis in an animal model [36]. Besides, WC is an indicator of abdominal fat accumulation, which is a risk factor to develop metabolic alterations such as insulin resistance, hyperinsulinemia, or T2DM [37]. Therefore, this study highlights the importance to have and maintain an optimal dietary $\omega 6: \omega 3$ PUFA ratio in order to improve metabolic profile.

The limitations of this study include its small sample size. Although only the dietary $\omega-6$ and $\omega$-3 PUFAs were evaluated, studies have demonstrated a strong correlation between the reported dietary and serum levels of these PUFAs [38]. Studies that measure dietary and plasma $\omega-6$ and $\omega-3$ PUFAs at the same time would help to clarify these results. Likewise, studies with a larger sample size to verify a possible causative effect of the dietary $\omega-6: \omega-3$ PUFA ratio on anthropometric parameters of adiposity and other adipocytokines are also necessary to clarify these associations.

\section{Conclusions}

This study suggests that a high dietary $\omega-6: \omega-3$ PUFA ratio is positively associated with measures of adiposity such as WC. In addition, subjects with higher dietary $\omega-6: \omega-3$ PUFA ratio had worse metabolic profile, specifically regarding insulin and HOMA-IR values. These findings could contribute to the design of more specific nutritional interventions in patients with high adiposity and insulin resistance.

\section{Funding}

This study was supported financially by grants from CONACyT (Salud-2013-01-202540).

\section{Authors' Contributions}

EML and IHC designed the study. NTC, JASG, WCP, EMBC, CABG, and KGB collected and processed the data. NTC, JASG, WCP, EMBC, and KGB analyzed the data. NTC, JASG, WCP, YFMS, IHC, MGC, and EML participated in data interpretation and manuscript preparation. 
Torres-Castillo et al.: High Dietary $\omega-6: \omega-3$ PUFA Ratio Is Positively Associated with Excessive Adiposity and Waist Circumference

\section{Disclosure Statement}

The authors have no conflicts of interest to disclose.

\section{References}

1 World Health Organization: Obesity and overweight. Geneva, World Health Organization. www.who.int/mediacentre/factsheets/fs311/en/ (last accessed August 3, 2018).

2 Barquera S: Encuesta Nacional de Salud y Nutrición 2016:resultados nacionales. (Instituto Nacional de Salud Pública, Mexico, editors). Cuernavaca, Instituto Nacional de Salud Pública, Secretaría de Salud, 2012, pp 68.

3 World Health Organization: Diet, Nutrition and the Prevention of Chronic Diseases. Report of a Joint WHO/ FAO Expert Consultation. WHO Technical Report Series 916, Geneva, World Health Organization, 2003. http:// apps.who.int/iris/bitstream/handle/10665/42665/WHO_TRS_916.pdf;jsessionid=40853001F466CF1B280510 413EBC5AF1?sequence=1 (last accessed August 3, 2018).

4 World Health Organization: Projections of Mortality and Causes of Death, 2015 and 2030. www.who.int/ healthinfo/global_burden_disease/projections/en/ (last accessed August 3, 2018).

5 Milic M, Frustaci A, Del Bufalo A, Sánchez-Alarcón J, Valencia-Quintana R, Russo P, Bonassi S: DNA damage in non-communicable diseases: a clinical and epidemiological perspective. Mutat Res Mol Mech Mutagen 2015; 776:118-127.

6 Barb D, Williams CJ, Neuwirth AK, Mantzoros CS: Adiponectin in relation to malignancies: a review of existing basic research and clinical evidence. Am J Clin Nutr 2007;86:858S-866S.

7 Ziemke F, Mantzoros CS: Adiponectin in insulin resistance: lessons from translational research. Am J Clin Nutr 2010;91:258S-261S

8 Bays HE, Toth PP, Kris-Etherton PM, Abate N, Aronne LJ, Brown WV, Gonzalez-Campoy JM, Jones SR, Kumar R, La Forge R, Samuel VT: Obesity, adiposity, and dyslipidemia: a consensus statement from the National Lipid Association. J Clin Lipidol 2013;7:304-383.

9 Cocate PG, Pereira LG, Marins JC, Cecon PR, Bressan J, Alfenas RC: Metabolic responses to high glycemic index and low glycemic index meals: a controlled crossover clinical trial. Nutr J 2011;10:1.

10 Enos RT, Velázquez KT, McClellan JL, Cranford TL, Walla MD, Murphy EA: Reducing the dietary omega6:omega-3 utilizing $\alpha$-linolenic acid; not a sufficient therapy for attenuating high-fat-diet-induced obesity development nor related detrimental metabolic and adipose tissue inflammatory outcomes. PLoS One 2014; 9:e94897.

11 Ramos LF, Shintani A, Ikizler TA, Himmelfarb J: Oxidative Stress and inflammation are associated with adiposity in moderate to severe CKD. JASN 2008;19:593-599.

12 Lorente-Cebrián S, Costa AG, Navas-Carretero S, Zabala M, Martínez JA, Moreno-Aliaga MJ: Role of omega-3 fatty acids in obesity, metabolic syndrome, and cardiovascular diseases: a review of the evidence. J Physiol Biochem 2013;69:633-651.

13 Simopoulos AP: An increase in the omega-6/omega-3 fatty acid ratio increases the risk for obesity. Nutrients 2016;8:128

14 Simopoulos AP: Omega-6/omega-3 essential fatty acid ratio and chronic diseases. Food Rev Int 2004;20: 77-90.

15 World Medical Association Declaration of Helsinki: Recommendations guiding physicians in biomedical research involving human subjects. JAMA 1997;277:925-926.

16 Bergman RN, Stefanovski D, Buchanan TA, Sumner AE, Reynolds JC, Sebring NG, Xiang AH, Watanabe RM: A better index of body adiposity. Obesity (Silver Spring) 2011;19:1083-1089.

17 Okosun IS, Liao Y, Rotimi CN, Choi S, Cooper RS: Predictive values of waist circumference for dyslipidemia, type 2 diabetes and hypertension in overweight White, Black, and Hispanic American adults. J Clin Epidemiol 2000;53:401-408.

18 Friedewald WT, Levy RI, Fredrickson DS: Estimation of the concentration of low-density lipoprotein cholesterol in plasma, without use of the preparative ultracentrifuge. Clin Chem 1972;18:499-502.

19 Roberts WC: The Friedewald-Levy-Fredrickson formula for calculating low-density lipoprotein cholesterol, the basis for lipid-lowering therapy. Am J Cardiol 1988;62:345-346.

20 Matthews DR, Hosker JP, Rudenski AS, Naylor BA, Treacher DF, Turner RC: Homeostasis model assessment: insulin resistance and beta-cell function from fasting plasma glucose and insulin concentrations in man. Diabetologia 1985;28:412-419.

21 Zhu N, Pankow JS, Ballantyne CM, Couper D, Hoogeveen RC, Pereira M, Duncan BB, Schmidt MI: High-molecularweight adiponectin and the risk of type 2 diabetes in the ARIC Study. J Clin Endocrinol Metab 2010;95:50975104.

22 Ramírez-Silva I, Villalpando S, Moreno-Saracho JE, Bernal-Medina D: Fatty acids intake in the Mexican population. Results of the National Nutrition Survey 2006. Nutr Metab (Lond) 2011;8:33.

23 Simopoulos AP: Evolutionary aspects of diet and essential fatty acids. World Rev Nutr Diet 2001;88:18-27.

24 Simopoulos AP: The importance of the omega-6/omega-3 fatty acid ratio in cardiovascular disease and other chronic diseases. Exp Biol Med (Maywood) 2008;233:674-688. 
Torres-Castillo et al.: High Dietary $\omega-6: \omega-3$ PUFA Ratio Is Positively Associated with Excessive Adiposity and Waist Circumference

25 Saltiel AR, Reilly SM: Adapting to obesity with adipose tissue inflammation. Nat Rev Endocrinol 2017;13:633.

26 Yamashita T, Oda E, Sano T, Yamashita T, Ijiru Y, Giddings JC, Yamamoto J: Varying the ratio of dietary n-6/n-3 polyunsaturated fatty acid alters the tendency to thrombosis and progress of atherosclerosis in apoE-/LDLR-/- double knockout mouse. Thromb Res 2005;116:393-401.

27 Liu H-Q, Qiu Y, Mu Y, Zhang X-J, Liu L, Hou X-H, Zhang L, Xu XN, Ji AL, Cao R, Yang RH, Wang F: A high ratio of dietary n-3/n-6 polyunsaturated fatty acids improves obesity-linked inflammation and insulin resistance through suppressing activation of TLR4 in SD rats. Nutr Res 2013;33:849-858.

28 Burns T, Maciejewski SR, Hamilton WR, Zheng M, Mooss AN, Hilleman DE: Effect of omega-3 fatty acid supplementation on the arachidonic acid:eicosapentaenoic acid ratio. Pharmacotherapy 2007;27:633-638.

29 Bagga D, Capone S, Wang HJ, Heber D, Lill M, Chap L, Glaspy JA: Dietary modulation of omega-3/omega-6 polyunsaturated fatty acid ratios in patients with breast cancer. J Natl Cancer Inst 1997;89:1123-1131.

30 Mohammadi E, Rafraf M, Farzadi L, Asghari-Jafarabadi M, Sabour S: Effects of omega-3 fatty acids supplementation on serum adiponectin levels and some metabolic risk factors in women with polycystic ovary syndrome. Asia Pac J Clin Nutr 2012;21:511-518.

31 Itoh M, Suganami T, Satoh N, Tanimoto-Koyama K, Yuan X, Tanaka M, Kawano H, Yano T, Aoe S, Takeya M, Shimatsu A, Kuzuya H, Kamei Y, Ogawa Y: Increased adiponectin secretion by highly purified eicosapentaenoic acid in rodent models of obesity and human obese subjects. Arterioscler Thromb Vasc Biol 2007;27:19181925.

32 Lund A-SQ, Hasselbalch AL, Gamborg M, Skogstrand K, Hougaard D.M, Heitmann BL, Kyvik KO, Sørensen TIA, Jess T: N-3 polyunsaturated fatty acids, body fat and inflammation. Obes Facts 2013;6:369-379.

33 Navas-Carretero S, San-Cristobal R, Avellaneda A, Planes J, Zulet MA, Martínez JA: Benefits on body fat composition of isocalorically controlled diets including functionally optimized meat products: role of alpha-linolenic acid. J Funct Foods 2015;12:319-331.

34 Mirmiran P, Hosseinpour-Niazi S, Naderi Z, Bahadoran Z, Sadeghi M, Azizi F: Association between interaction and ratio of $\omega-3$ and $\omega-6$ polyunsaturated fatty acid and the metabolic syndrome in adults. Nutrition 2012;28: 856-863.

35 Hennig B, Watkins BA: Linoleic acid and linolenic acid: effect on permeability properties of cultured endothelial cell monolayers. Am J Clin Nutr 1989;49:301-305.

36 Ukropec J, Reseland JE, Gasperikova D, Demcakova E, Madsen L, Berge RK, Rustan AC, Klimes I, Drevon CA Sebökova E: The hypotriglyceridemic effect of dietary n-3 FA is associated with increased beta-oxidation and reduced leptin expression. Lipids 2003;38:1023-1029.

37 Klein S, Allison DB, Heymsfield SB, Kelley DE, Leibel RL, Nonas C, et al: Waist circumference and cardiometabolic risk: a consensus statement from Shaping America's Health: Association for Weight Management and Obesity Prevention; NAASO, The Obesity Society; the American Society for Nutrition; and the American Diabetes Association. Diabetes Care 2007;30:1647-1652.

38 Amiano P, Dorronsoro M, de Renobales M, Ruiz de Gordoa JC, Irigoien I; EPIC Group of Spain: Very-long-chain omega-3 fatty acids as markers for habitual fish intake in a population consuming mainly lean fish: the EPIC cohort of Gipuzkoa. European Prospective Investigation into Cancer and Nutrition. Eur J Clin Nutr 2001;55: 827-832. 